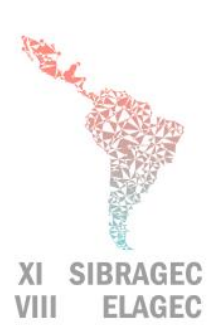

\title{
HEPO- SYSTEM OF FEEDBACK OF BUILDINGS FROM REAL CONDITIONS OF USE
}

\author{
RESTREPO ÁLVAREZ, Mario Fernando (1); VÁSQUEZ HERNÁNDEZ, \\ Alejandro (2)
}

(1)Universidad EAFIT, +(57) 3015456806, e-mail: mrestr16@eafit.edu.co (2) Universidad EAFIT, email: avasqu12@eafit.edu.co

\begin{abstract}
The evaluation of buildings in real conditions of use or post-occupancy evaluation, is the systematic study of buildings with the objective of decide their performance once they are inhabited; is a process of review and identification of successes and failures, in order to improve the conditions of existing buildings and to feed future designs, in search of residential satisfaction. Nowdays, the construction industry in Colombia does not have feedback processes regarding the operation of a building; Therefore, the research presented below proposes a tool for post-occupational evaluation-HEPO-, which is oriented to the analysis of buildings in use phase, in terms of meeting needs and preserving quality in the time of the materials, providing as a result the variables of study for the Colombian sector, in order to understand the performance dynamics, and proposes the mechanisms of collection and processing the information for the correct presentation of results, also presents the barriers at the time of HEPO application. In order to verify the effectiveness of the tool, it is validated in five housing projects in the city of Medellin, Colombia.
\end{abstract}

Keywords: Post-occupancy evaluation, Sustainability, Occupant satisfaction, Conservation of qualities in time, Occupancy Comfort

\section{INTRODUCCIÓN}

El sector de la construcción posee falencias en cuanto al conocimiento que tiene del funcionamiento de las edificaciones, esto se debe a que no cuenta con procesos de retroalimentación que permitan identificar y evaluar los aciertos y desaciertos de su producto. La estrategia utilizada para predecir el comportamiento de una edificación es la modelación; con esta se pretende desde la fase de diseño simular condiciones de uso. Aun así, no necesariamente los modelos planteados para dichas simulaciones corresponden con lo que realmente se presenta en fase de uso, encontrando diferencias sustanciales entre las suposiciones consideradas en la modelación y el comportamiento real de las edificaciones (OLIVIA et al.,2015), las cuales, en algunos casos, en relación al recurso energético, se expresan en consumos reales de más de tres veces la cantidad predicha en la modelación (BORDASS et al., 2004)

En muchas ocasiones, el mal funcionamiento en los proyectos de vivienda colectiva en altura se relacionan con el desconocimiento que se tiene en cuanto al comportamiento en condiciones reales de uso, generalmente por falencias relacionadas con la 
satisfacción de las necesidades de los usuarios finales (LEAMAN et al.,2010), como lo afirma (ADESOJI, 2012) cuando asegura que "el fracaso recurrente de proyectos de vivienda se debe a la falta de información y lecciones aprendidas derivadas de la perspectiva de los usuarios finales". Otra de las falencias se da en términos de las conservaciones de las calidades en el tiempo, ya que algunas edificaciones evidenciaban costos de mantenimiento muy superiores a lo presupuestado, debido al rápido deterioro de los materiales (LEAMAN et al., 2010).

Por lo anterior, POE puede ser definido como:

“...el estudio sistemático de edificaciones con el objetivo de determinar su desempeño una vez estas se encuentran habitadas, en un proceso de revisión que permite extraer enseñanzas a partir de la identificación y análisis de aciertos y desaciertos, con la finalidad de mejorar las condiciones de edificaciones existentes y retroalimentar futuros diseños" (RESTREPO et al., 2017)

A nivel global, se muestran que Reino Unido, EE. UU., China, Australia, Canadá y Malasia son más activos en la investigación de POE, mientras que en Latinoamérica el país con más avance en Brasil (PEIXIAN LI et al., 2018) evidenciándose de manera clara la necesidad de contar con un instrumento de evaluación Post Ocupacional para el territorio colombiano, que permita extraer aciertos y desaciertos del sector inmobiliario.

\section{METODOLOGÍA}

La investigación presentada tuvo como objetivo el desarrollo de una herramienta de evaluación post ocupacional (HEPO), con la finalidad de analizar las edificaciones en condiciones reales de uso. Para ello se realizaron las siguientes acciones para llegar al éxito del desarrollo de la herramienta.

\subsection{Desarrollo de la Herramienta de Evaluación (HEPO)}

Se toma como punto de partida la fundamentación referencial sobre lo que ha marcado la tendencia en las evaluaciones de edificaciones en condiciones de uso a nivel mundial, y el estudio de las variables contempladas, las herramientas de levantamiento de la información utilizadas y los tipos de evaluación post ocupacional existente.

\subsection{Validación de la Clasificación}

Se llevaron a cabo entrevistas con expertos para discutir y mejorar la herramienta propuesta. El objetivo del ejercicio fue recabar las impresiones y observaciones que los expertos tenían, al respecto del contenido de la herramienta en función a los factores de evaluación como de la forma de recopilación de los datos.

\subsection{Validación con caso de estudio}

Se seleccionó una muestra de cinco proyectos, no bajo parámetros de rigurosidad estadística sino con base en la rigurosidad descriptiva establecida por los siguientes parámetros: temporalidad, estrato socioeconómico, tipología de la vivienda, vinculación con el entorno cercano, y por último el sistema estructural. 
SIBRAGEC - ELAGEC 2019 - del 23 al 25 de Octubre - LONDRINA - PR

\section{HERRAMIENTA DE EVALUACIÓN POST OCUPACIONAL (HEPO)}

\subsection{Estado del arte}

En la revisión bibliográfica se encontró que los factores de evaluación de interés durante la fase de uso de las edificaciones son 37, siendo los más estudiados el confort térmico (XUE et al., 2016; ZALEJSKA, 2014), acústico (ADEWUNMI et al., 2012); lumínico (BONDE et al., 2015) y calidad del aire interior (HASSANAIN, 2016). El porcentaje de aparición de cada uno de los aspectos en las investigaciones abordadas es presentado en la Figura 1.

\section{Figura 1 - Factores de estudio en edificaciones en condiciones de uso}

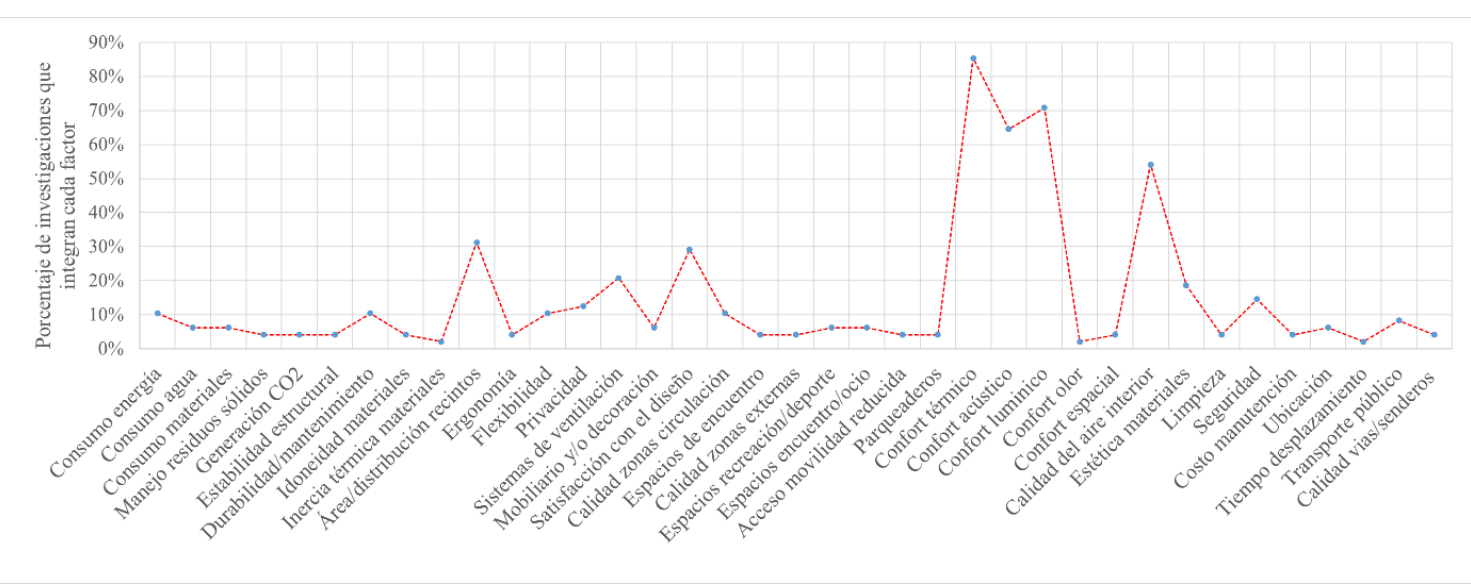

Fuente: RESTREPO et al., (2017).

Para recopilar información sobre el funcionamiento de las edificaciones en condiciones reales de uso, normalmente se acude a las herramientas de percepción, monitoreo y herramientas de observación.

Las metodologías de evaluación post-ocupacional pretenden, en la medida de lo posible, abordar un mismo factor desde herramientas de recolección de información relacionadas con varios de estos grupos, con la intención de contrarrestar la información resultante de la aplicación de diversas herramientas y obtener un conocimiento más amplio.

Dependiendo de la intención y objeto de la investigación post ocupacional, los factores de estudio abordados son analizados con diferentes niveles de profundidad $\mathrm{y}$ rigurosidad, Preiser (WOLFGANG, 1995) y Turpin-Brooks (TURPIN et al., 2006) identificaron tres tipos de evaluación post ocupacional, que tienen directa relación con el nivel de profundidad del estudio realizado: indicativo, investigativo y diagnóstico.

\subsubsection{Escala de evaluación de HEPO}

Determinar el funcionamiento de una edificación requiere de la compresión del proceso habitacional, ya que la vivienda no es solamente el espacio que se encuentra de la puerta de ingreso hacia adentro, sino además aquellos espacios más allá de su espacio privado, como es el caso de corredores, zonas de diversión y encuentro tanto del conjunto residencial como de su entorno inmediato. Por lo cual, es necesario analizar las diferentes escalas de una vivienda, y estas son las mostradas en la Tabla 1. 
SIBRAGEC - ELAGEC 2019 - del 23 al 25 de Octubre - LONDRINA - PR

Tabla 1 - Escala de evaluación HEPO.

\begin{tabular}{|l|l|}
\hline Escala de evaluación & \multicolumn{1}{|c|}{ Componentes de la escala } \\
\hline Espacio privado & $\begin{array}{l}\text { Recientes que conforman la unidad habitacional, tales como: } \\
\text { comedor, habitaciones, baño, etc. }\end{array}$ \\
\hline Espacio semiprivado & $\begin{array}{l}\text { Zonas comunes dentro de una misma torre, tales como: } \\
\text { corredores y escalera. }\end{array}$ \\
\hline Espacio semipúblico & $\begin{array}{l}\text { Espacios comunes dentro del conjunto residencial pero al } \\
\text { exterior de las torres, tales como: parques, lobby, porteria, etc. }\end{array}$ \\
\hline Espacio público & $\begin{array}{l}\text { Entornos inmediatos conformados por vías, tales como: } \\
\text { equipamientos públicos, parques, entre otros. }\end{array}$ \\
\hline
\end{tabular}

Fuente: Elaboración propia

\subsubsection{Estrategias de recolección de la información}

Se plantean 3 estrategias que permitan recabar la información deseada de la edificación, la primera de ellas, las encuestas de percepción; para tal actividad se aplicó la metodología de Likert, una estrategia que evalúa actitudes y opiniones; Observación, por lo cual se hacen recorridos por el conjunto habitacional con la finalidad de identificar aciertos y desaciertos en cuanto al funcionamiento de la edificación; y por último el Monitoreo, de las variables en estudio buscando confrontar las percepciones que los usuarios brindan sobre dichos aspectos.

\subsubsection{Herramienta de evaluación post ocupacional (HEPO)}

Una vez definidos los ejes de evaluación, la escala de interés y las herramientas para el levantamiento de la información de HEPO, se presenta en la Figura 2 la relación entre estas variables. 
SIBRAGEC - ELAGEC 2019 - del 23 al 25 de Octubre - LONDRINA - PR

Figura 2 - Herramienta de evaluación Post Ocupacional (HEPO).

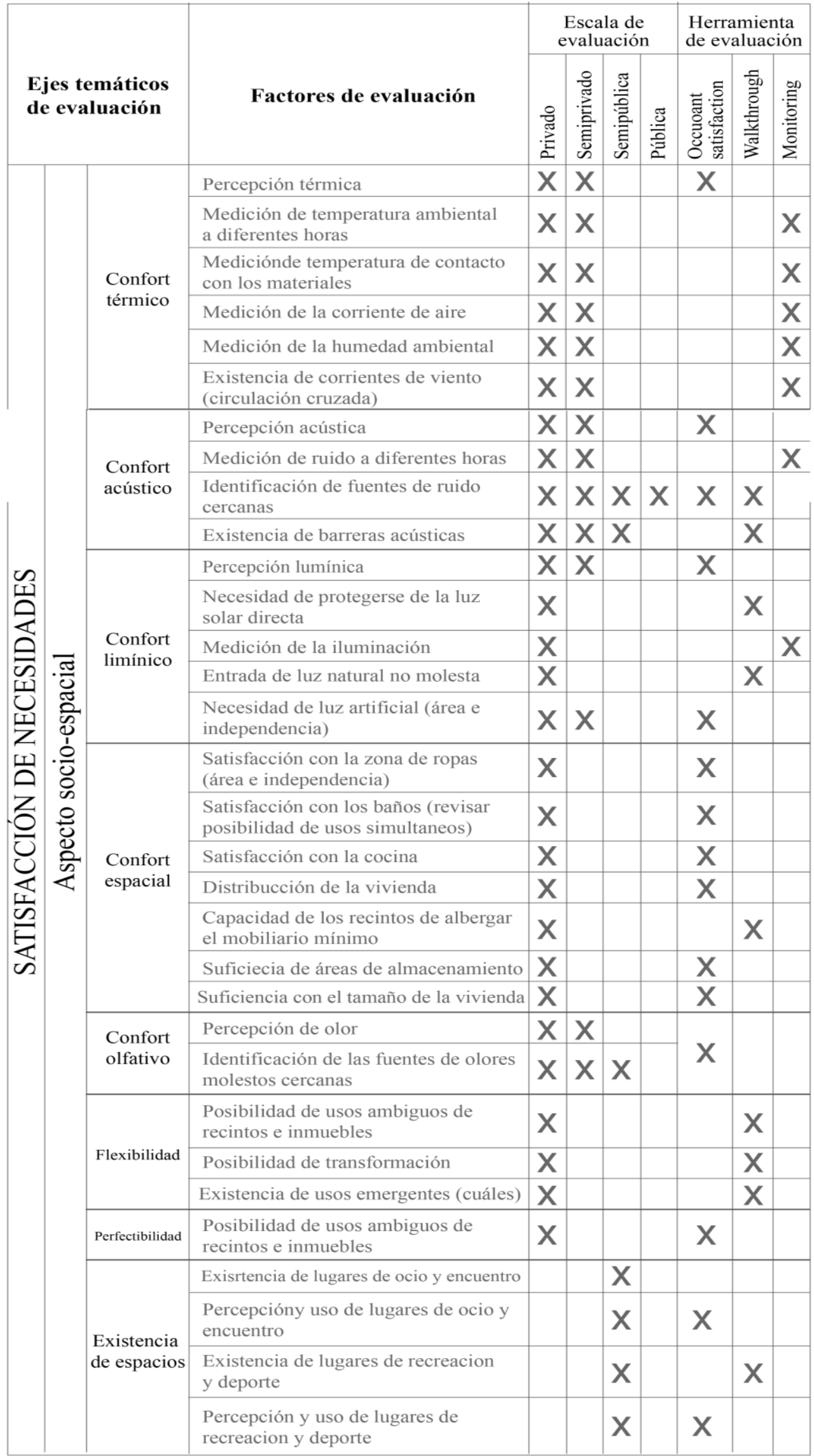


SIBRAGEC - ELAGEC 2019 - del 23 al 25 de Octubre - LONDRINA - PR

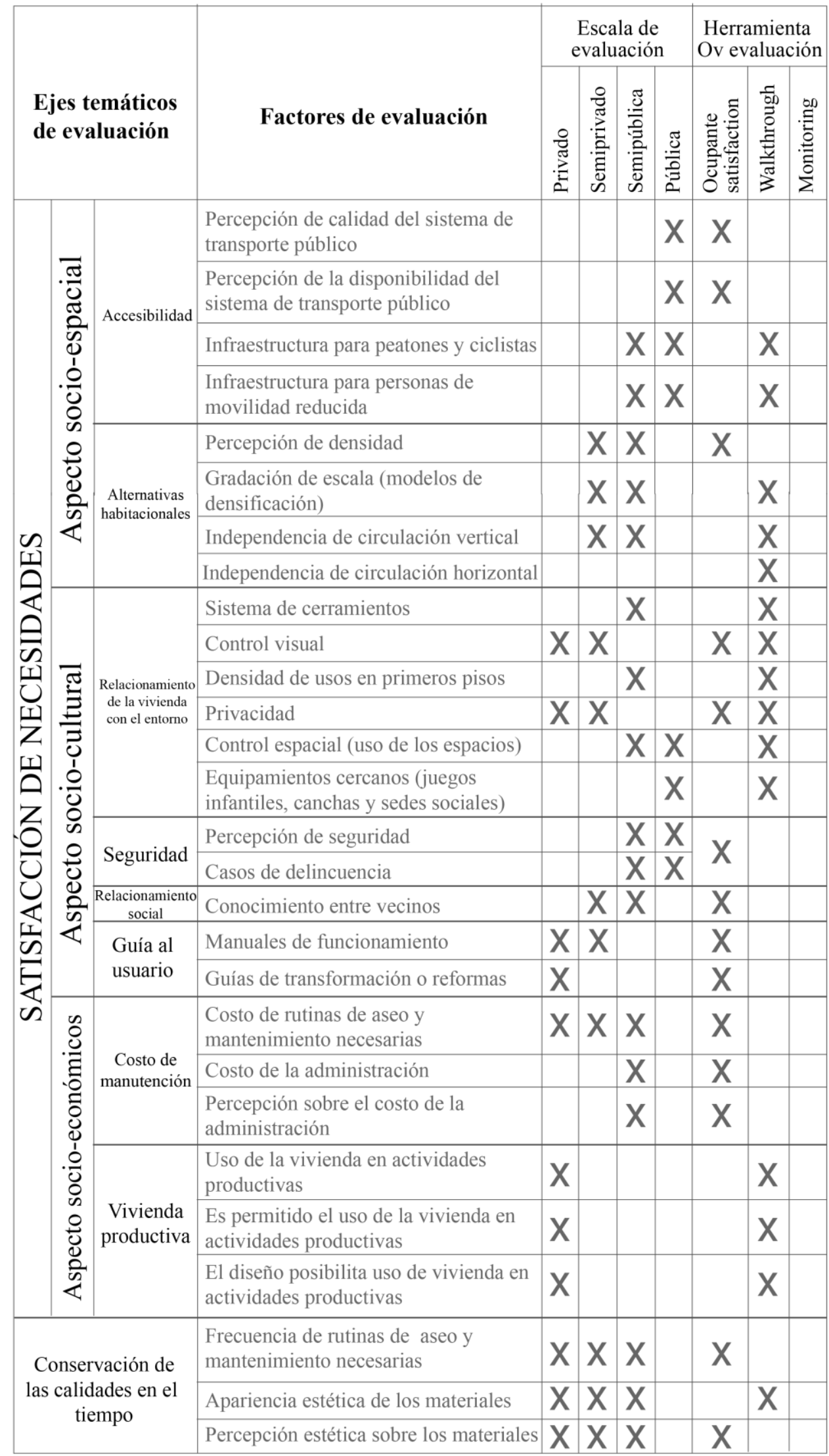

Fuente: Elaboración propia 
SIBRAGEC - ELAGEC 2019 - del 23 al 25 de Octubre - LONDRINA - PR

\subsection{Validación a partir del caso de estudio}

HEPO permite recolectar dos tipos de información, desarrolla el cálculo de índices de satisfacción por factor de estudio e índice de satisfacción residencial y evalúa la conservación de las condiciones de las calidades de los materiales a través del tiempo.

\subsubsection{Satisfacción de necesidades}

Ya que los datos adquiridos con las encuentas de percepción son recabados estableciendo una escala de satisfacción de 1 a 5, HEPO determina un índice de satisfacción (IS) por factor de evaluación, sabiendo que, la clasificación de satisfacción y los pesos dan como resultado una ponderación que da cuenta del grado de satisfacción de los residentes. Los valores IS calculados en HEPO tienen como base la fórmula planteada por Mohammad et al., (2010).

$$
\text { Satisfation Index }(S I)=\frac{\sum_{i=1}^{5}(a i)(x i)}{5 \sum_{i=1}^{5}(x i)} * 100 \%
$$

Donde, $\quad$ ai $=$ peso asignado a cualquier indicador;

$\mathrm{xi}=$ frecuencia relativa asignada a cualquier indicador.

La respuesta para (i) es 1, 2, 3, 4 y 5, siendo 1 el adjetivo negativo (Muy insatisfecho) y 5 el positivo (Muy satisfecho).

- SI entre 0-50\%, implica que los ocupantes están muy insatisfechos.

- SI entre 51-60\%, implica que los ocupantes están insatisfechos.

- SI entre 61-80\%, implica que los ocupantes tienen una opinión neutra

- SI entre $81-90 \%$, implica que los ocupantes están satisfechos.

- SI entre 91-100\%, implica que los ocupantes están fuertemente satisfechos.

La Figura 3 es la forma como HEPO presenta los indices de satisfacción para cada uno de los factores de evaluación integrados a la herramienta.

Figura 3 - Percepción de bienestar en la vivienda.

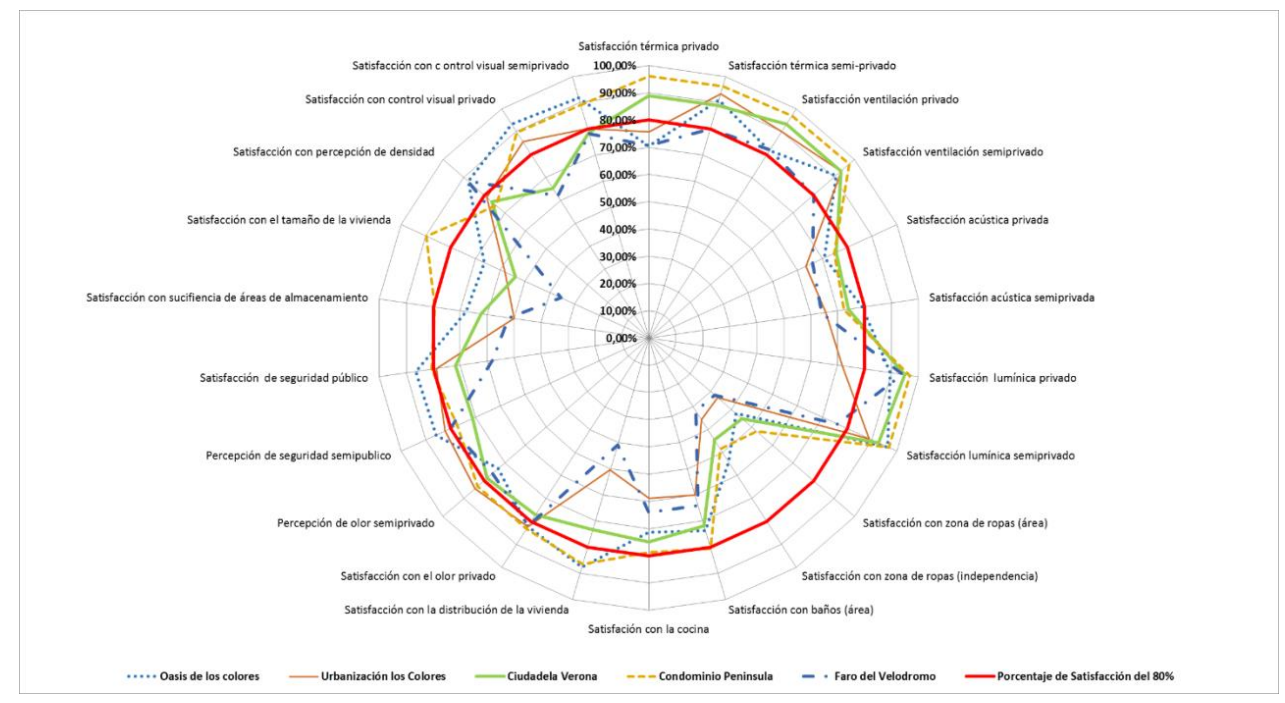

Fuente: Elaboración propia 
Asimismo, HEPO determina el indice de satisfacción residencial, que no es más que la suma total de los índices de satisfacción de componentes y es calculada con la ecuación propuesta por Mohammad et al., (2010).

$$
S I r=\frac{\sum_{i=1}^{N 1} d i+\sum_{i=1}^{N 2} S i+\sum_{i=1}^{N A} p i+\sum_{i=1}^{N 4} S e i+\sum_{i=1}^{N 5} n i}{\sum_{i=1}^{N 1} D i+\sum_{i=1}^{N 2} S i+\sum_{i=1}^{N 3} P i+\sum_{i=1}^{N 4} S E i+\sum_{i=1}^{N 5} N i} \times 100
$$

donde, SIr = índice de satisfacción residencial;

$\mathrm{N} 1, \mathrm{~N} 2, \mathrm{~N} 3, \mathrm{~N} 4$ y N5 = número de variables seleccionadas para escalar en cada componente de la vivienda;

SI, pi, sei y NI = puntuación real de un encuestado en la ia variable en el componente;

Di, Si, Pi, SEi y Ni = máximos posibles calificaciones de la ia variable evaluada. A partir de lo anterior, se obtienen valores como los presentados en la Tabla 2.

Tabla 2 - Índice de satisfacción residencial

\begin{tabular}{|c|c|c|c|c|c|}
\hline $\begin{array}{c}\text { Conjunto } \\
\text { residencial }\end{array}$ & $\begin{array}{c}\text { Oasis de los } \\
\text { colores }\end{array}$ & $\begin{array}{c}\text { Urbanización } \\
\text { los colores }\end{array}$ & $\begin{array}{c}\text { Ciudadela } \\
\text { Verona }\end{array}$ & $\begin{array}{c}\text { Condominio } \\
\text { Península }\end{array}$ & $\begin{array}{c}\text { Faro del } \\
\text { Velódromo }\end{array}$ \\
\hline Sir & $78.80 \%$ & $70.92 \%$ & $74.80 \%$ & $85.75 \%$ & $66.24 \%$ \\
\hline
\end{tabular}

Fuente: Elaboración propia

HEPO además permite la adquisición de respuestas abiertas que posibiliten la identificación de causas de insatisfacción, con miras a procesos de retroalimentación.

\subsubsection{Conservación de las calidades en el tiempo}

Evaluar la conservación de las calidades en el tiempo es un aspecto nuevo en las evaluaciones post ocupacionales realizadas hasta ahora, es por lo anterior que HEPO aborda este factor por la importancia que tiene saber cuál es el comportamiento de los materiales y cómo esto afecta el funcionamiento de las edificaciones, en aspectos como comportamiento de los materiales ante agentes atmosféricos específicos de un lugar, ante la interacción con fauna y flora propia del lugar, posibles causas de deterioro acelerado, rutinas reales de mantenimiento preventivo y correctivo necesarias, dificultad de rutinas de aseo y costos asociados, comportamiento del material ante intervenciones domésticas menores realizadas por parte de los habitantes en el proceso de apropiación, facilidad de reparación de deterioros menores, y consumo de recursos asociado al mantenimiento en el tiempo del material.

\section{BARRERAS PARA LA IMPLEMENTACIÓN DE HEPO}

Existen varios elementos que generan barreras para la implementación de la herramienta HEPO, estas se clasifican de la siguiente forma: 
SIBRAGEC - ELAGEC 2019 - del 23 al 25 de Octubre - LONDRINA - PR

Figura 4 - Barreras para la implementación.

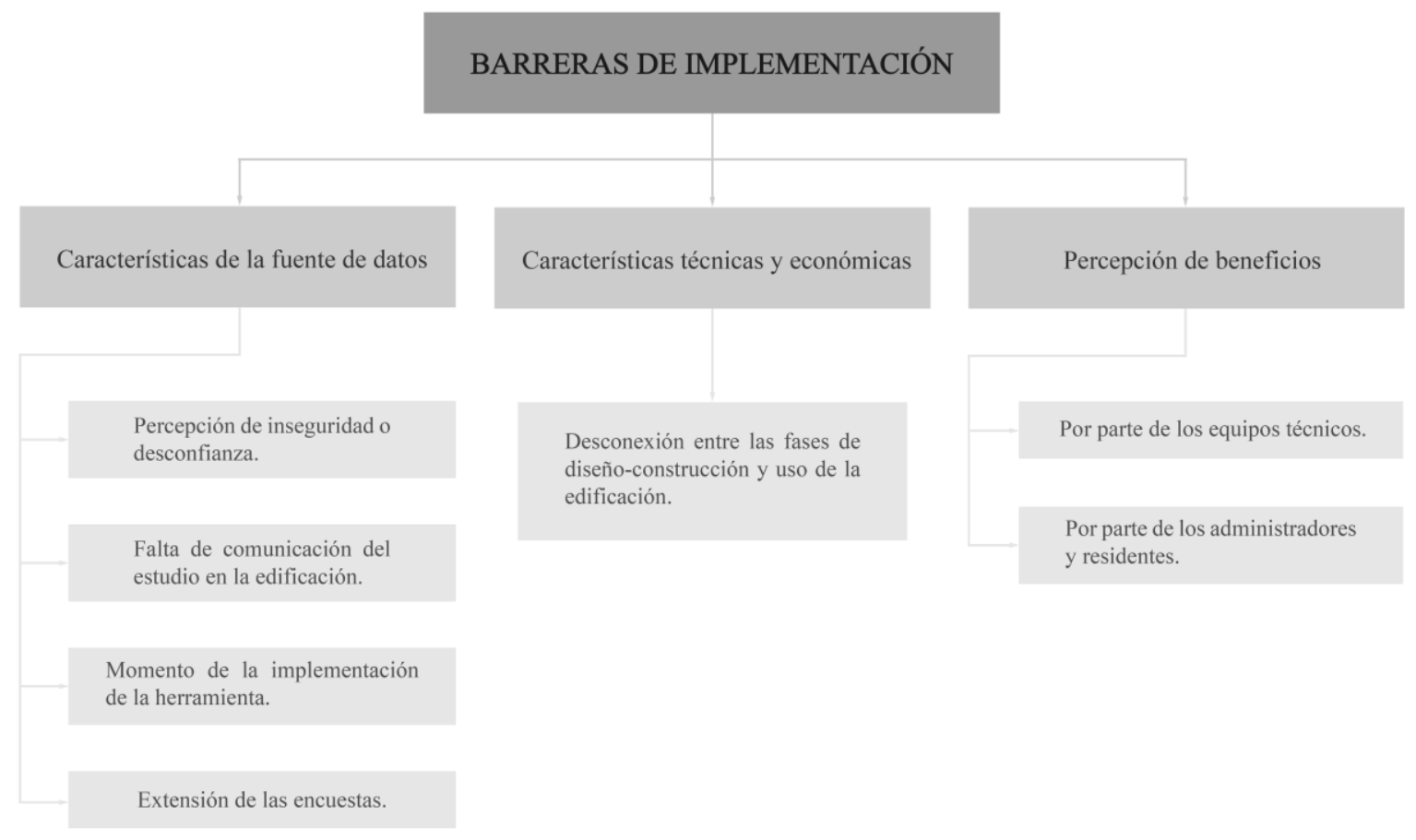

Fuente: Elaboración propia

\section{CONCLUSIONES}

El desarrollo de estrategias de retroalimentación es importante para mejorar las condiciones de vida de los residentes ya que pone en evidencia las correspondencias y discordancias entre lo diseñado y el espacio habitado, pero debido a la falta de integración entre las fases de una edificación, muchos proyectos de POE están limitados en términos de vincular su evaluación entre estas, asimismo identifica claramente las posibilidades que se tienen para que los espacios se adecuen de mejor manera a las necesidades.

Ningún protocolo estandarizado de POE ha ganado dominio mundial o nacional, lo anterior es posiblemente atribuido a las condiciones inherentes de cada país para desarrollar sus edificaciones, es por esto la necesidad de conocer y entender nuestro entorno para asi formular una estrategia de evaluación, que no dependa de los casos de estudio, sino por el contrario generar patrones de analisis generales para las edificaciones.

\section{REFERENCIAS}

ADESOJI, DAVID JIBOYE, A. Post-occupancy evaluation of residential satisfaction in Lagos, Nigeria: Feedback for residential improvement. Frontiers of Architectural Research, v. 1, n. 3, p. 236-243, 2012.

LEAMAN, A., STEVENSON, F.; BORDASS, B. Building evaluation: practice and principles. Building Research \& Information, p. 564-577, 2010. 
TURPIN-BROOKS, S.; VICCARS, G. The development of robust methods of post occupancy evaluation. Facilities, v. 24, n. 5/6, p. 177-196. 2006

VÁSQUEZ-HERNÁNDEZ, ALEJANDRO; RESTREPO ÁLVAREZ, M. F. Evaluation of buildings in real conditions of use_Revisado. Journal of Building Engineering, v. 12, p. 2636. 2017.

WOLFGANG, P. Post-occupancy evaluation: how to make buildings work better. Facilities, v. 13, n. 11, p. 19-28. 1995.

OLIVIA, C. G. S.; \& AIDAN, T. In-use monitoring of buildings: An overview and classification of evaluation methods. Energy and Buildings, v. 86, p. 176-189. 2015.

XUE, P.; MAK, C. M.; CHEUNG, H. D.; \& CHAO, J. Post-occupancy evaluation of sunshades and balconies' effects on luminous comfort through a questionnaire survey. Building Services Engineering Research \& Technology, v. 37, n. 1, p. 51-65. 2016.

ZALEJSKA-JONSSON, A. Parameters contributing to occupants' satisfaction. Facilities, v. 32, n. 7/8, p. 411-437. 2014.

BORDASS, B.; COHEN, R.; FIELD, J. Energy performance of non-domestic buildings: closing the credibility gap. BUILDING PERFORMANCE CONGRESS, 1-10, 2004.

ADEWUNMI, YEWANDE; OMIRIN, MODUPE; FAMUYIWA, F. Post-occupancy evaluation of postgraduate hostel facilities. Facilities, v. 29, n. 3/4, p. 149-168. 2012.

BONDE, M.; \& RAMIREZ, J. A post-occupancy evaluation of a green rated and conventional on-campus residence hall. International Journal of Sustainable Built Environment, v. 4, n. 2, p. 400-408. 2015.

HASSANAIN, M. A. Post-Occupancy Indoor Environmental Quality Evaluation of Student Housing Facilities. Architectural engineering and design management, v. 3, n. 4, p. 249 256. 2016.

PEIXIAN, LI.; THOMAS, M. FROESE.; GAIL, BRAGER. Post-occupancy evaluation: Stateof-the-art analysis and state-of-the-practice review. Building and Environment, v. 133, p. 187202. 2018.

MOHAMMAD, ABDUL MOHIT; MANSOR, IBRAHIM; YONG, RAZIDAH RASHID. Assessment of residential satisfaction in newly designed public low-cost housing in Kuala Lumpur, Malaysia. Habitat International. v. 34, p. 18-27. 2010. 